\title{
NEOPLASIAS DUODENAIS PRIMÁRIAS: ANÁLISE DE CASUÍSTICA E CONDUTA CIRÚRGICA
}

\author{
PRIMARY DUODENAL TUMORS: CLINICOPATHOLOGIC EXPERIENCE AND \\ SURGICAL TREATMENT
}

\author{
Sansom Henrique Bromberg, ACBC-SP ${ }^{1}$ \\ Paulo Schmidt Goffi Júnior, TCBC-SP ${ }^{2}$ \\ Fábio Schmidt Goffi, ECBC-SP ${ }^{3}$ \\ Nagamassa Yamaguchi, TCBC-SP ${ }^{4}$ \\ Edson Ussami ${ }^{5}$ \\ Erasmo de Magalhães Tolosa ${ }^{6}$
}

\begin{abstract}
RESUMO: São apresentados 18 casos de neoplasias primárias do duodeno, salientando-se sua raridade e as dificuldades diagnósticas, a despeito dos modernos recursos endoscópicos e de identificação por imagem. A literatura revela que de $1 \%$ a $10 \%$ de todos os tumores do aparelho digestório se situam no intestino delgado. A localização duodenal é, por isso, muito pouco freqüente, possui sintomatologia indefinida e implica conduta terapêutica mais complexa do que quando o tumor se situa em outros segmentos do intestino delgado. Os sintomas mais comuns são dor, náusea, vômito e hemorragia. A presente casuística consiste de 11 neoplasias malignas e sete benignas. Entre as primeiras o adenocarcinoma é o mais freqüente, sendo os lipomas os mais comuns dos neoplasmas benignos. Enquanto nestes a exérese local representa a conduta mais adequada, a duodenopancreatectomia cefálica foi a cirurgia de escolha nos tumores malignos da segunda porção duodenal, exibindo bons resultados. As lesões malignas da terceira porção e principalmente da quarta porção do duodeno foram tratadas pela ressecção duodenojejunal, também com resultados satisfatórios.
\end{abstract}

Unitermos: Neoplasias duodenais. Tratamento cirúrgico. Duodenopancreatectomia.

\section{INTRODUÇÃO}

A literatura médica revela que apenas $1 \%$ a $10 \%$ de todos os tumores malignos do sistema digestório se situam no intestino delgado ${ }^{1-3}$, em que pese sua extensão anatômica e a situação entre dois segmentos - estômago e cólons -, sedes freqüentes de lesões malignas.

Em decorrência, é rara a localização duodenal de neoplasias, cuja sintomatologia é indefinida e a conduta terapêutica pode ser mais complexa do que quando o tumor se encontra em outros segmentos do tubo digestório. A origem embrionária do duodeno, conjunta com as vias biliares e o pâncreas, que alguns denominaram "simbiose biliopancreatoduodenal", e suas relações sintópicas com estruturas vasculares importantes, tornam difíceis as manobras de dissecção e manipulação cirúrgicas. Resulta que a cirurgia radical dos tumores bilioduodenopancreáticos é mais invasiva.

Há poucas contribuições sobre o tema publicadas por autores nacionais e, mesmo essas, se referem a escassa casuística $^{3-5}$. Na literatura internacional encontram-se relatos exibindo maior experiência, mas também aqui predominam as apresentações de casos isolados ${ }^{6-10}$.

1. Doutor em Medicina da UNIFESP. Vice-Coordenador de Pós-Graduação em Gastroenterologia Cirúrgica do IAMSPE

2. Professor Assistente Doutor FM-USP. Assistente da Divisão de Clínica Cirúrgica do Hospital Universitário- USP

3. Professor Emérito FM-USP. Presidente da Comissão de Pós-Graduação do IAMSPE-HSPE

4. Professor Assistente Doutor FM-USP. Assistente do Serviço de Gastroenterologia Cirúrgica do HSPE

5. Assistente da Divisão de Clínica Cirúrgica do Hospital Universitário - USP

6. Professor Titular do Departamento de Cirurgia da FM-USP

Recebido em 17/9/99

Aceito para publicação em 4/4/2000

Trabalho dos Serviços de Gastroenterologia Cirúrgica e Anatomia Patológica do HSPE-FMO e do Hospital Universitário da Universidade de São Paulo 
Por isso, justifica-se a revisão da nossa experiência colhida no Hospital do Servidor Público Estadual FMO e no Hospital Universitário da Universidade de São Paulo.

\section{PACIENTES E MÉTODOS}

Colecionamos 18 casos de neoplasias duodenais primárias não papilares, dos quais 12 são originários do Serviço de Gastroenterologia Cirúrgica do Hospital do Servidor Público Estadual, três são oriundos do Setor de Clínica Cirúrgica do Hospital Universitário da Universidade de São Paulo e três de necropsia pertencem ao Serviço de Anatomia Patológica do Hospital do Servidor Público Estadual.

As lesões malignas somaram 11 casos e as benignas se manifestaram sete vezes - quatro achados cirúrgicos casuais e três observações de necropsia. Dez pacientes eram do sexo masculino e oito pertenciam ao sexo feminino. As idades variaram de 18 a 77 anos, sendo a mediana de 53 anos para os pacientes com tumores malignos e de 71 para os benignos.

Os pacientes com doença benigna eram assintomáticos e as lesões foram surpreendidas no decurso de gastroduodenectomia por úlcera péptica ou de duodenotomia para exploração da papila duodenal maior.

Nos portadores de blastomas duodenais malignos os sintomas caracterizaram-se, invariavelmente, por dor abdominal, náuseas e vômitos, enquanto que o emagrecimento manifestou-se em 55,6\% dos pacientes (5/9). Uma doente com carcinóide ulcerado do duodeno, além dos comemorativos apontados, apresentava diarréia, hemorragia gastrointestinal e rubor (flushing) cutâneo intermitente. O sangramento gastrointestinal também esteve presente em outra paciente com linfoma ulcerado da segunda porção duodenal.

Das 11 lesões malignas, seis localizavam-se na segunda porção do duodeno, quatro na terceira ou quarta porção e uma (carcinóide) estava situada no bulbo duodenal. Constavam de seis adenocarcinomas, dos quais um papilífero, dois carcinóides, um linfoma, um carcinoma indiferenciado e um carcinoma neuroectodérmico. As neoplasias duodenais benignas eram, na presente casuística, quatro lipomas, dois pólipos adenomatosos e um leiomioma.
Foram praticadas cinco gastroduodenopancreatectomias cefálicas (GDPC) em tumores da segunda porção duodenal, uma GDPC em tumor da terceira porção, três ressecções parciais duodenojejunais ( RDJ) em lesões da terceira e quarta porções e uma gastroduodenectomia (GD) em paciente portador de úlcera gástrica, tendo sido encontrada pequena lesão carcinóide $(1 \mathrm{~cm} \times 1 \mathrm{~cm})$ no bulbo duodenal ressecado. Um enfermo com carcinoma indiferenciado inextirpável da segunda porção duodenal foi submetido, apenas, à biópsia e à gastrojejunostomia (GJ) (Tabela 1).

\section{RESULTADOS}

Os seis pacientes com neoplasia maligna não papilar (cinco da segunda porção e um da terceira porção do duodeno), tratados pela GDP, tiveram alta hospitalar em bom estado, sendo acompanhados por períodos que variaram de 18 meses a oito anos, com mediana de quatro anos. Houve seguimento de três deles, respectivamente, durante dois, seis e oito anos, depois do que se perderam. Ocorreu recidiva em outros dois pacientes 18 meses e um ano após a cirurgia. $\mathrm{O}$ último submetido à GDPC, acompanhado por sete anos após a cirurgia, apresentou nova lesão maligna, representada por carcinoma epidermóide do esôfago, vindo a falecer por disseminação dessa doença logo após seu diagnóstico.

Os três doentes tratados pela RDJ também tiveram alta hospitalar em boas condições, sendo acompanhados por 16 meses, dois e quatro anos. O primeiro deles depois se perdeu, o segundo faleceu com recidiva e o último faleceu de infarto agudo do miocárdio.

O portador de carcinoma indiferenciado do duodeno, submetido apenas à biópsia e à gastrojejunostomia, teve alta hospitalar mas faleceu no período pós-operatório imediato. Finalmente, perdeu-se o controle após a alta hospitalar do doente em que foi surpreendido, casualmente, um pequeno tumor carcinóide $(1 \mathrm{~cm} \times 1 \mathrm{~cm})$ do bulbo duodenal na peça resultante de GD por úlcera gástrica.

Nos enfermos com neoplasias duodenais benignas, como já reportado, as lesões foram encontradas na vigên-

Tabela 1

Neoplasias duodenais malignas

\begin{tabular}{|c|c|c|c|c|c|c|c|c|}
\hline Paciente & Hospital & Diagnóstico & Local & Tamanho & Cirurgia & Sobrevida & Evolução & Causa \\
\hline AS & HSPE & $\mathrm{ADCa}$ & $4^{\mathrm{a}} \mathrm{P}$ & $4,5 \times 3,5$ & RDJ & $4^{\mathrm{a}}$ & óbito & IAM \\
\hline $\mathrm{HC}$ & HSPE & $\mathrm{ADCa}$ & $4^{\mathrm{a}} \mathrm{P}$ & $4,0 \times 3,0$ & RDJ & $2^{\underline{a}}$ & óbito & Recid. \\
\hline CPM & HSPE & $\mathrm{ADCa}$ & $2^{\underline{a}} \mathrm{P}$ & $6,5 \times 4,5$ & GDP & $6^{\mathrm{a}}$ & extrav. & - \\
\hline MLM & HSPE & $\mathrm{ADCa}$ & $3^{\mathrm{a}} \mathrm{P}$ & $4,0 \times 3,0$ & RDJ & $16 \mathrm{~m}$. & extrav. & - \\
\hline $\mathrm{AMC}$ & HSPE & Linfoma & $2^{\mathrm{a}} \mathrm{P}$ & $3,5 \times 3,0$ & GDP & $1 \mathrm{a}$ & óbito & Recid \\
\hline HG & HSPE & ADCa papilífero & $2 \underline{\mathrm{a}}$ & $3,0 \times 3,0$ & GDP & $8^{\mathrm{a}}$ & extrav. & - \\
\hline MGL & $\mathrm{HU}$ & Carcinóide & $2^{\mathrm{a}} \mathrm{P}$ & $3,0 \times 3,0$ & GDP & $2^{\underline{a}}$ & extrav. & - \\
\hline JB & HSPE & Carcinóide & $1^{\mathrm{a}} \mathrm{P}$ & $1,0 \times 1,0$ & exérese & - & - & - \\
\hline $\mathrm{RS}$ & HSPE & Ca Indiferenc. & $3^{\mathrm{a}} \mathrm{P}$ & - & Biópsia derivaç. & $30 \mathrm{~d}$ & óbito & Toxem \\
\hline JGA & HU & Ca neuroectod. & $2^{\mathrm{a}} \mathrm{P}$ & $2,0 \times 1,0$ & GDP & $18 \mathrm{~m}$ & óbito & Recid. \\
\hline GD & $\mathrm{HU}$ & $\mathrm{ADCa}$ & $3^{\mathrm{a}} \mathrm{P}$ & $1,5 \times 1,5$ & GDP & $7^{\mathrm{a}}$ & óbito & Ca ep. Esof. \\
\hline
\end{tabular}

$\mathrm{ADCa}=$ adenocarcinoma $\mathrm{Ca}=$ carcinoma $\mathrm{P}=$ porção; $\mathrm{RDJ}=$ ressecção duodenojejunal; $\mathrm{GDP}=$ gastroduodenopancreatectomia; Recid. $=$ recidiva $;$ Extrav. $=$ extraviado $;=$ anos; m.= meses; Ca ep. Esof. = Carcinoma epidermóide do esôfago. 
cia de operação indicada para tratamento de outras afecções e, por isso, não tiveram seguimento pós-operatório específico.

\section{DISCUSSÃO}

O tratamento radical das lesões duodenais malignas implica cirurgia complexa, mais agressiva do que a realizada para ressecar as demais neoplasias do intestino delga$\mathrm{do}^{11}$. Resulta que o diagnóstico dos tumores duodenais deve ser preciso e precoce a fim de propiciar a conduta cirúrgica mais adequada.

Os sintomas causados pelos blastomas duodenais malignos são escassos, traduzidos por dor, geralmente relacionada com episódios de obstrução ou suboclusão ${ }^{1}$. Paralelamente manifestam-se náuseas, vômitos e anorexia.

A presença do neoplasma determina perda do peso corpóreo por efeitos metabólicos, e, principalmente, porque compromete o livre trânsito alimentar.

Conquanto alguns autores afirmem ser comum a hemorragia digestiva maciça, ela só foi anotada em dois de nossos casos. Eram pacientes com tumores da segunda porção, sendo um linfoma e um carcinóide, este caso também acompanhado de diarréia e rubor.

A duodenografia contrastada e a gastroduodenoscopia constituem recursos práticos e úteis para o diagnóstico. No entanto, as lesões da terceira e quarta porções do duodeno nem sempre são percebidas pelo estudo radiológico e o endoscópio habitual não alcança essas porções. A tomografia computadorizada pode ser de valia para a detecção e estadiamento dos tumores extraluminares.

A biópsia peroperatória para surpreender o caráter maligno do achado, tem valor relativo e depende, entre outros fatores, da experiência do patologista e da qualidade da amostra encaminhada. Em nossa paciente com adenocarcinoma papilífero da segunda porção duodenal este exame mostrou-se falso negativo. Por isso, a melhor conduta é a cirurgia radical representada pela GDPC adotada no caso em apreço com bons resultados. Até porque a degeneração maligna pode ocorrer em $26 \%$ a $72 \%$ das lesões vilosas ${ }^{12,13}$.

A mesma opção é válida para as lesões carcinóides do duodeno, em que as exéreses locais conduzem a reiteradas recidivas ${ }^{14}$, sendo a GDPC a conduta mais adequada ${ }^{4,8,10}$.

Os adenocarcinomas do duodeno são raros. No entanto, eles figuram como os mais freqüentes tumores duodenais ${ }^{1,15}$. Por outra parte, se localizam muito mais vezes na segunda do que na terceira e quarta porções ${ }^{16}$. Quase sempre são diagnosticados em estádio adiantado de desenvolvimento, uma vez que as lesões superficiais, não infiltrando a submucosa e ditas "precoces" passam despercebidas e, por isso, poucas vezes são relatadas 5 . Parece haver risco maior do aparecimento de câncer duodenal em pacientes com adenomatose familiar cólica e adenoma do duodeno ${ }^{15,17,18}$.

A literatura consigna que as idades mais atingidas pelo carcinoma duodenal estão na década dos $60 \operatorname{anos}^{16}$. Na presente casuística os pacientes tinham progressivamente 29 , 43, 53, 59, 72 e 72 anos.

Os sintomas causados pelos neoplasmas eram vagos e traduzidos por dor, vômitos e emagrecimento, o que está de acordo com os informes da literatura ${ }^{19}$. Nenhum de nossos pacientes apresentou icterícia, que é relatada em até $54 \%$ dos enfermos ${ }^{16,19,20}$. Apenas a portadora de adenocarcinoma viloso da segunda porção teve surtos de pancreatite aguda, sem icterícia.

Os pacientes que sofreram cirurgia radical, tanto a GDPC quanto a RDJ tiveram alta hospitalar em bom estado e sobrevida compensatória. A GDPC, não obstante seu vulto, é o melhor tratamento para as neoplasias da segunda porção duodenal, opinião que é corroborada pela maioria dos autores $2,7,17,19,20,21$. Mesmo a presença de metástases linfonodais parece não ter impacto na sobrevida dos enfermos e não deve contra-indicar a ressecção ${ }^{2,23}$. A GDPC, assim, deve ser o procedimento de escolha no tratamento dos adenocarcinomas do duodeno, sempre que tecnicamente exeqüível e que as condições clínicas permitirem, ainda que na presença de metástases linfonodais regionais ${ }^{20}$.

Nossa experiência confirma a opinião da literatura de que os pacientes com adenocarcinoma do duodeno têm mais probabilidade de conseguir sobrevida maior após GDPC do que os portadores de outras formas de adenocarcinoma periampular ${ }^{24}$.

Os resultados das RDJ foram também satisfatórios a curto e médio prazos, não se justificando a conduta conservadora $^{2}$, como a simples gastrojejunostomia, no tratamento das neoplasias malignas ressecáveis da terceira e quarta porções duodenais.

As lesões duodenais benignas são raras, assintomáticas $^{1}$ e constituem habitualmente achados cirúrgicos ou de necropsia. São lipomas, pólipos adenonomatosos, leiomiomas que devem ser identificados para não serem confundidos com tumores malignos ${ }^{25}$. Seu tratamento limita-se à ressecção local ${ }^{1}$.

\begin{abstract}
Eighteen primary duodenal tumors, including 11 malignant and 8 benign, are presented, stressing their poorly defined natural history and rare frequency. The most common histological diagnosis was adenocarcinoma. There were 6 adenocarcinomas, 2 carcinoids, 1 linfoma, 1 anaplastic and 1 neuroectodermic carcinoma. Benign lesions were occasionally found during a gastroduodenal or a biliary surgery $(n=4)$ and during the autopsies $(n=3)$. They consisted of lipomas $(n=4)$, adenomatous polyps $(n=2)$ and leiomyoma $(n=1)$. Concerning the malignant lesions, 6 pancreaticoduodenectomies, 3 duodenojejunal segmentary resection and one paliation consisting of gastrojejunostomy and biopsy were performed. One carcinoid lesion was found at the duodenal bulb during a gastroduodenectomy for a gastric ulcer. Pancreaticoduodenectomy can be done with acceptable risk and it seems
\end{abstract}


that patients with duodenal adenocarcinoma are more likely to survive longer after radical surgery than those with cancer of the head of pancreas. Every patient of this series treated by pancreaticoduodenectomy or duodenojejunal segmentary resection had an uneventful recovery and the follow-up ranged from 16 months to 7 years. As for the duodenal carcinoids, local resection is usually followed by recurrence, so an agressive surgical management is the best approach. Benign tumors must be treated by local excision when disclosed.

Key words: Duodenal cancer. Treatment. Small intestinal tumors.

\section{REFERÊNCIAS}

1. Kaminski N, Shahan D, Eliakim R, et al. - Primary tumours of the duodenum. Posgrad Med J 1993; 69: 136-8.

2. Rose DM, Hochwald SN, Klimstra DS, et al. - Primary duodenal adenocarcinoma: ten- year experience with 79 patients. J Amer Coll Surg 1996; 183: 89-96.

3. Souza HP, Toneto MC, BOnfanti A, et al. - Adenocarcinoma primário do duodeno. Rev Col Bras Cir 1999; 26: 124.

4. Goffi FS, Goffi Junior PS, Schwartz RW, et al. - Recurrent carcinoid of the small intestine. Posgrad Gen Surg 1990; 2: 84-8.

5. Jukemura J, Amico EC, Penteado S, et al. - Adenocarcinoma precoce de duodeno. Relato de caso. Rev Hosp Clin Fac Med Univ S Paulo 1996; 5: 258-60.

6. Cleator IGM - Carcinoma of the third part of the duodenum. Brit J Surg 1970; 57: 934-7.

7. Edman P - Duodenal cancer. Report of a case. Acta Chir Scand 1973; 139: 759-62.

8. Jones MA, Griffith JM, West AB - Adenocarcinoid tumor of periampullary region: a novel duodenal neoplasm presenting as biliary tract obstruction. Human Path 1989; 20: $198-200$.

9. Yildirin S, Culhaoglu AB, Ozdemir N - Carcinoma of the forth part of the duodenum: report of a case. Surg Today 1995; 25: 1034-7.

10. Rämö OJ, Risthari SKK, Miettinen M, et al. - Duodenal carcinoid tumour with posammoma bodies: a rare cause of haematemesis. Eur J Surg 1997; 163: 227-30.

11. Goffi FS, Bromberg SH, Silva LC, et al. - Duodenopancreatectomias: indicações, técnica e resultados. Análise de 54 casos. Rev Ass Med Bras 1979; 24:381-4.

12. Rosenberg J, Welch JP, Pyrtek LJ, et al. - Benign villous adenomas of the ampulla of Vater. Cancer 1986; 58:1563-8

13. Gibbs ER, Walton GF, Kent III RB, et al. - Villous tumors of the ampulla Vater. Am Surg 1997; 63:467-71.

14. Sakorafas GH, Sarr MG, Tsiotos GG, et al. - Villous tumors of the duodenum: reappraisal of local versus extended resection. Gastroenterology; 1999, 116:A1310 [abstract S0069].

15. Williamson R - Primary carcinoma of the duodenum: analysis of survival. Invited commentary. World J Surg 1988;12:699.
16. Scott-Coombes DM, Williamson RCN - Surgical treatment of primary duodenal carcinoma; a personal series. Brit J Surg 1994; 81: 1472-4.

17. Adedeji AO, Trescoli-Serrano C, Garcia-Zarco M - Primary duodenal carcinoma. Posgrad Med J 1995; 71:354-8.

18. Wallace MH, Phillips RK - Upper gastrointestinal disease in patients with familiar adenomatous poliposis. Brit $J$ Surg 1998; 85:742-50.

19. Lai ECS, Doty JE, Irving C, et al. - Primary adenocarcinoma of the duodenum: analysis of survival. World JS 1988; 12:695-9.

20. Rotman N, Pezet D, Fagniez PL, et al. - Adenocarcinoma of the duodenum: factors influencing survival. Brit J Surg 1994; 81:83-5.

21. Box JC, Watne AL, Lucas GW - Small bowel carcinoid: review of a single institution experience and review of the literature. Am Surg 1996; 62:280-6.

22. Stephens J, Kuhn J, O'Brien J, et al. - Surgical morbidity, mortality and long-term survival in patients with peripancreatic cancer following pancreaticoduodenectomy. Am J Surg 1887; 174: 600-3.

23. Pickleman J, Koelsh M, Cheifec G - Node-positive duodenal carcinoma Is Curable. Arch Surg 1997; 132: 241-4.

24. Yeo CJ, Sohn TA, Cameron JL et al. - Periampullary adenocarcinoma: analysis of 5-year survivors. Ann Surg 1998; 227: 821-31.

25. Moyana TN, Miller GG, Keith RG - Sporadic ampullary hamartoma simulating cancer. JCC, 1997; 40: 227-30.

\section{ENDEREÇO PARA CORRESPONDÊNCIA:}

Dr. Sansom Henrique Bromberg

Inst. Assist. Médica Serv. Pub. Estadual

Divisão de Ensino e Pesquisa

Av. Ibirapuera, 981

04029-000 — São Paulo-SP 